\title{
A coupling strategy between the BEM and Kansa's method for acoustic analysis of heterogeneous media
}

\author{
L. Godinho \& A. Tadeu \\ CICC-Research Centre on Construction Science, \\ Department Civil Engineering, University of Coimbra Rua Luis Reis \\ Santos, Portugal
}

\begin{abstract}
The BEM is well established as a solid numerical technique for the analysis of acoustic and wave propagation problems. Its numerical formulation relies on previous knowledge of the Green's functions for the specific problem to be studied, and thus it is usually limited to homogeneous media or to media incorporating regions with piecewise constant or with well-defined variations (such as linearly varying) of properties. In fact, the definition of these Green's functions becomes difficult whenever more generic cases are considered, such as when the properties of the propagation media vary from point to point. For this case, when the properties of the medium inside a sub-region exhibit spatial variations, alternative approaches have to be used. In this work, a frequency domain model based on the joint use of the BEM and of the Kansa's method is proposed to model such systems. In that model, the BEM is used to model the homogeneous part of the propagation domain, while Kansa's method is used to model an heterogeneity, which may exhibit velocity variations from point to point. In the interface between the two parts of the domain, continuity of velocities and pressures are imposed. Kansa's method is applied here making use of Multiquadric (MQ) RBFs, which incorporate a shape parameter that strongly influence the quality of its results. To overcome this problem, a general strategy based on the assessment of the domain error in the enforcement of the governing PDE is introduced to allow the definition of adequate values of this shape parameter.
\end{abstract}

Keywords: boundary elements, Kansa's method, acoustics, coupled methods. 


\section{Introduction}

Wave propagation in fluids and solids is a subject of interest to many branches of engineering sciences, such as acoustics, geophysics or oceanography. During the last two decades, the boundary element method (BEM) has become one of the major numerical techniques used for sound propagation analysis, particularly in domains involving unbounded or semi-infinite media [1-3]. Its main advantage over other well-established methods, such as the FEM and FDM, is that it can solve the wave equation requiring only the discretization of the boundary interfaces. However, it involves a sophisticated mathematical formulation, and requires the prior knowledge of fundamental solutions, which are available only for some specific scenarios. Its efficiency also depends on the special treatment of numerical integration of the singular and hyper-singular integrals.

Mainly in the last decade, meshless methods have been progressively developed for different fields of applied physics. One such technique is the radial basis function (RBF) collocation method (Kansa's method [4, 5]). Kansa's method is based on the use of RBFs to describe the pressure field within a specific sub-domain, and so it does not require the use of Green's functions. Although different studies have been published on the use of this technique in acoustics, they are mostly restricted to solving the Helmholtz equation in problems involving $2 \mathrm{D}$ and $2.5 \mathrm{D}$ domains composed of homogeneous subdomains (see, for example, Tadeu et al. [6]).

In many problems, the domain cannot be assumed as perfectly homogeneous, and the most common configurations correspond to inhomogeneous media, either with a random or with a structured spatial or even temporal variation. From a physical point-of-view, the sound propagation velocity within a fluid varies with different factors, such as temperature and pressure, as well as with the presence of dissolved or suspended particles or chemicals within the propagation medium. If this variation is random, the variability of the propagation domain originates complex patterns with amplitudes and phase variations showing a random behaviour. For this reason, these types of problems are best described using statistical averages and probability densities. An overview of classical methods that can be used to assess the behaviour of these systems can be found in the works of Ishimaru [7].

As can be inferred from above, it is, in many cases, desirable to model propagation velocities' variations in a more detailed form, allowing for the simulation of inhomogeneous media. In this work, a numerical frequency domain model based on the joint use of the BEM and of Kansa's method is applied to model such systems. The BEM is used to model the homogeneous part of the propagation domain, while Kansa's method is used to model a heterogeneity, with smooth velocity variations from point to point. In the interface between the two parts of the domain, continuity of velocities and pressures is imposed. The method is formulated and validated against analytical solutions known for simple geometries. Kansa's method is here applied making use of Multiquadric (MQ) RBFs, which incorporate a shape parameter that strongly influence the quality of its results. To overcome this problem, a general 
strategy based on the assessment of the domain error in the enforcement of the governing PDE is introduced to allow definition of adequate values of this shape parameter.

\section{Mathematical formulation}

\subsection{Governing PDE}

In the frequency domain, the acoustic pressure field $(p(\mathrm{x}), \mathrm{x}$ being a generic domain point with coordinates $(x, y))$ within a heterogeneous fluid medium with varying propagation velocity $\alpha(\mathrm{x})$, and assuming null initial conditions, can be described by the governing partial differential equation

$$
\nabla^{2} p(\mathrm{x})+\frac{\omega^{2}}{\alpha(\mathrm{x})^{2}} p(\mathrm{x})=0,
$$

$\omega$ being the frequency, in $\mathrm{rad} / \mathrm{s}$.

Analytical solution of this equation can be written in an explicit form only in a limited number of situations, mainly in the presence of simple geometries. However, the analysis of complex domain, with internal boundaries and varying properties, can be performed using different numerical methods. Below, two numerical methods are described, namely the BEM, which can be used for piecewise constant media with well-defined interfaces, and the RBF collocation method (usually designated as Kansa's method), which allows considering generic point to point property variations.

\subsection{The RBF collocation method (Kansa's method)}

Consider a closed domain $\Omega$, bounded by a boundary $\Gamma$. Within this domain, consider a set of $N$ collocation points $\left\{\left(x_{k}, y_{k}\right)\right\}_{k=1}^{N}$, of which $\left\{\left(x_{k}, y_{k}\right)\right\}_{k=1}^{N I}$ are $N I$ internal points and $\left\{\left(x_{k}, y_{k}\right)\right\}_{k=N I+1}^{N}$ are boundary points. This distribution is illustrated in fig. 1 .

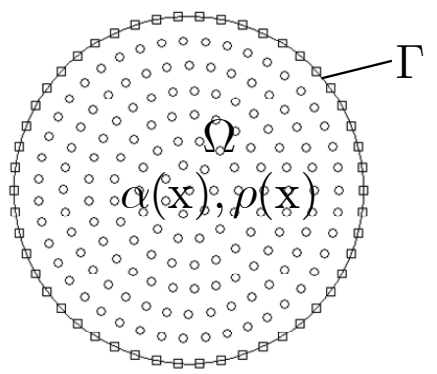

Figure 1: Illustration of node distribution for Kansa's method. Circles represent internal nodes and squares represent boundary nodes. 
In Kansa's method, the exact solution, $p(\mathrm{x})$, in eqn. (1), is approximated as $\hat{p}(\mathrm{x})$ using a linear combination of Radial Basis Functions (RBFs). This combination can be expressed as

$$
\hat{p}(\mathrm{x})=\sum_{k=1}^{N} a_{k} \varphi_{k}(\mathrm{x})
$$

where $\varphi_{k}(\mathrm{x})$ is the used RBF and $\left(a_{k}\right)_{k=1}^{N}$ are the $N$ unknown coefficients to be determined. Substituting $p(\mathrm{x})$ by $\hat{p}(\mathrm{x})$, and considering eqn. (2), eqn. (1) becomes

$$
\nabla^{2} \sum_{k=1}^{N} a_{k} \phi_{k}(\mathrm{x})+\frac{\omega^{2}}{\alpha(\mathrm{x})^{2}} \sum_{k=1}^{N} a_{k} \phi_{k}(\mathrm{x})=0
$$

Using this approximation, applying eqn. (3) at each internal point and imposing adequate boundary conditions at each boundary point (in terms of pressure, particle velocity or a combination of both), it becomes possible to establish a system of $N$ equations on $N$ unknowns, which allows calculation of the $\left(a_{k}\right)_{k=1}^{N}$ coefficients.

After calculation of those coefficients, the approximate solution at any given domain point can be computed using eqn. (2). It is worth noting that, given the intrinsic characteristics of the method, it becomes very simple to ascribe varying physical properties (in this case varying velocities) from point to point, and thus the method is adequate for solving problems in non-homogeneous domains.

In spite of the large choice of RBFs, Multi-Quadric functions (MQ) are possibly the most widely adopted by researchers due to the accurate results they can provide. These functions are defined as

$$
\varphi_{k}(\mathrm{x})=\sqrt{r_{k}^{2}+c^{2}}
$$

with $r_{k}=\sqrt{\left(x-x_{k}\right)^{2}+\left(y-y_{k}\right)^{2}},\left(x_{k}, y_{k}\right)$ being the center of the RBF, and $c$ being a shape (or free) parameter of the RBF. In fact, the definition of this parameter is a key point in the definition of accurate models making use of these RBFs, since its value can greatly affect the accuracy of the solution. It is know that large values of the shape parameter lead to smoother functions, and can usually provide better solutions. However, those large values also lead to illconditioned equation systems, which can greatly deteriorate the quality of the obtained solutions. The definition of its "optimal" value is still a subject of research, without a definitive conclusion (see, for example, the works of Tadeu et al. [6] or Cheng et al. [8]). 


\subsection{The Boundary Element Method (BEM)}

Consider, now, an infinite, homogeneous domain $\left(\Omega_{2}\right)$, with density $\rho_{2}$ and allowing sound waves to travel with a velocity $\alpha_{2}$, and containing a generic inclusion, limited by a boundary $\Gamma$.

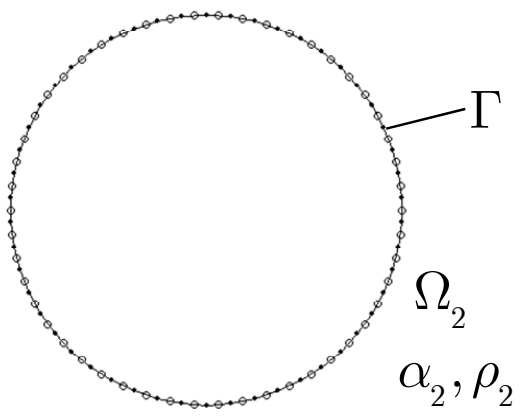

Figure 2: Illustrative representation of the BEM discretization. Open circles represent nodal points, while filled circles represent element limits.

In the absence of any distributed domain load, and considering a virtual unit point load oscillating with an angular frequency $\omega$ acting at point $\mathrm{x}_{0}$, the following boundary integral equation may be written:

$$
\begin{aligned}
c_{0} p\left(\mathrm{x}_{0}\right)= & -\mathrm{i} \rho_{2} \omega \int_{\Gamma} G\left(\mathrm{x}, \mathrm{x}_{0}\right) v(\mathrm{x}, \vec{n}) d \Gamma-\int_{\Gamma} H\left(\mathrm{x}, \mathrm{x}_{0}, \vec{n}\right) p(\mathrm{x}) d \Gamma \\
& +p_{\text {inc }}\left(\mathrm{x}_{0}, \mathrm{x}_{\mathrm{f}}\right)
\end{aligned}
$$

where $p$ and $v$ represent the pressure and the particle velocity, respectively, $G\left(\mathrm{x}, \mathrm{x}_{0}\right), H\left(\mathrm{x}, \mathrm{x}_{0}, \vec{n}\right)$ are, respectively, the Green's functions for pressure and its first spatial derivative along a direction $\vec{n}$, normal to the boundary and pointing outwards, corresponding to the response at a boundary point $\mathrm{x}$ due to a unit point pressure load applied at $\mathrm{x}_{0} ; p_{\text {inc }}\left(\mathrm{x}_{0}, \mathrm{x}_{\mathrm{f}}\right)$ represents the incident pressure field generated by a possible point load located within the domain at $\mathrm{x}_{\mathrm{f}} ; c_{0}$ is a constant which, for a smooth boundary, assumes the value $1 / 2$.

For an infinite fluid medium, the relevant Green's function can be defined by

$$
G\left(\mathrm{x}, \mathrm{x}_{0}\right)=-\frac{\mathrm{i}}{4} \mathrm{H}_{0}^{(2)}\left(\frac{\omega}{\alpha_{2}} \sqrt{\left(x-x_{0}\right)^{2}+\left(y-y_{0}\right)^{2}}\right)
$$

where $\mathrm{H}_{0}^{(2)}(\ldots)$ is the Hankel function of the second type and order 0 .

After discretizing the boundary $\Gamma$ in $N_{b e}$ segments (elements), equation (5) may be written as 
After discretizing the boundary $\Gamma$ in $N_{b e}$ segments (elements), equation (5) may be written as

$$
\begin{aligned}
c_{0} p\left(\mathrm{x}_{0}\right)= & -\mathrm{i} \rho_{2} \omega \sum_{j=1}^{N_{b e}} \int_{\Gamma_{j}} G\left(\mathrm{x}, \mathrm{x}_{0}\right) v(\mathrm{x}, \vec{n}) d \Gamma_{j}-\sum_{j=1}^{N_{b e}} \int_{\Gamma_{j}} H\left(\mathrm{x}, \mathrm{x}_{0}, \vec{n}\right) p(\mathrm{x}) d \Gamma_{j} \\
& +p_{\text {inc }}\left(\mathrm{x}_{0}, \mathrm{x}_{f}\right)
\end{aligned}
$$

Application of the load sequentially at all nodal points allows establishing $N_{b e}$ equations. The necessary integrations along the boundary can, in general, be performed by means of Gaussian quadrature method. However, in order to ensure a good the accuracy of the method, when the loaded element coincides with the integrated element, the resulting singular integration should be performed analytically.

\subsection{Coupling BEM with Kansa's method}

As stated before, one of the strongest limitations of the BEM is that it requires the previous knowledge of the Green's functions for the propagation medium, which can be difficult to obtain when the medium properties have generic spatial variations. The use of a coupled methodology making use of the BEM and of Kansa's method may then be a useful approach to model problems which include portions of the domain with variation of properties, since Kansa's method is particularly well suited to model those portions. This can be particularly interesting when the only small portions of the domain exhibit those variations of the propagation velocity, allowing the use of the BEM for the homogeneous part and of Kansa's method for the heterogeneous portion.

Given the mathematical formulation of both methods presented above, it becomes simple to establish a coupling strategy between both methods, using the BEM for the unbounded part, and Kansa's method for the localized portions with properties' variation. In what follow, the authors propose a direct coupling strategy between the two methods, which is valid only when the nodes used in the BEM model match the boundary nodes used for Kansa's method. Indeed, if the boundary nodes used for both methods coincide, it becomes possible to impose the continuity of the relevant quantities, namely pressure and particle velocity.

Considering the medium properties defined in the previous subsections, and imposing pressure and velocity continuity, the approximations given by Kansa's method for pressure and for velocity can be substituted in eqn. (7), which becomes

$$
\begin{aligned}
c_{0} p\left(\mathrm{x}_{0}\right)= & -\mathrm{i} \rho_{2} \omega \sum_{j=1}^{N_{b e}} \int_{\Gamma_{j}} G\left(\mathrm{x}, \mathrm{x}_{0}\right) \hat{v}(\mathrm{x}, \vec{n}) d \Gamma_{j}-\sum_{j=1}^{N_{b e}} \int_{\Gamma_{j}} H\left(\mathrm{x}, \mathrm{x}_{0}, \vec{n}\right) \hat{p}(\mathrm{x}) d \Gamma_{j}, \\
& +p_{\text {inc }}\left(\mathrm{x}_{0}, \mathrm{x}_{f}\right)
\end{aligned}
$$


or, writing those approximations in explicit form, and bearing in mind that

$$
\begin{aligned}
\hat{v}(\mathrm{x}, \vec{n})= & \frac{1}{-\mathrm{i} \rho \omega} \frac{\partial \hat{p}}{\partial \vec{n}}, \\
c_{0} p\left(\mathrm{x}_{0}\right)= & \frac{\rho_{2}}{\rho} \sum_{j=1}^{N_{b e}} \int_{\Gamma_{j}} G\left(\mathrm{x}_{\mathrm{x}_{0}}\right) \sum_{k=1}^{N} a_{k} \frac{\partial}{\partial \vec{n}} \phi_{k}(\mathrm{x}) d \Gamma_{j} \\
& -\sum_{j=1}^{N_{b e}} \int_{\Gamma_{j}} H\left(\mathrm{x}, \mathrm{x}_{0}, \vec{n}\right) \sum_{k=1}^{N} a_{k} \phi_{k}(\mathrm{x}) d \Gamma_{j}+p_{i n c}\left(\mathrm{x}_{0}, \mathrm{x}_{f}\right)
\end{aligned}
$$

Writing eqn. (9) for each of the nodal points of the BEM allows establishing $N_{b e}$ equations, all of them being a function of the $N$ unknowns $\left(a_{k}\right)_{k=1}^{N}$, which correspond to the unknown amplitudes of the RBFs. The remaining set of equations can be defined by establishing the governing equation (eqn. (3)) at each of the NI internal points defined for Kansa's method. After solving this $N \times N$ system, the $\left(a_{k}\right)_{k=1}^{N}$ amplitudes of the RBFs can then be determined.

The knowledge of those amplitudes then allows calculation of the nodal values of pressure and velocity along the boundary. At the nodal point $j$, those quantities can be computed as:

$$
\begin{gathered}
p\left(\mathrm{x}_{j}\right)=\sum_{k=1}^{N} a_{k} \phi_{k}\left(\mathrm{x}_{j}\right) \\
v\left(\mathrm{x}_{j}\right)=\sum_{k=1}^{N} a_{k} \frac{\partial}{\partial \vec{n}} \phi_{k}\left(\mathrm{x}_{j}\right)
\end{gathered}
$$

In the outer (unbounded) domain $\Omega_{2}$, the pressure at any domain point can then be calculated by making use of the standard integral equation, as given in eqn. (8).

\section{Evaluation of the error for the coupled solution}

To evaluate the accuracy of the proposed coupled method, consider the configuration depicted in fig. 3, corresponding to a host fluid medium, allowing a velocity of $1500 \mathrm{~m} / \mathrm{s}$, containing a circular inclusion with a radius of $1.0 \mathrm{~m}$ centred with the coordinated axis system, filled with a fluid that allows a propagation velocity of $1800 \mathrm{~m} / \mathrm{s}$. This system is illuminated by a $2 \mathrm{D}$ source placed at $(x=-5.0 \mathrm{~m} ; y=0.0 \mathrm{~m})$, which emits acoustic waves with a given frequency. For this case, it is possible to define a reference solution in a closed form following the strategy defined, for example, in Tadeu et al. [9]. To analyze the behavior of the numerical approach, consider a grid of receivers, distributed between $(x=-2.0 \mathrm{~m} ; y=-2.0 \mathrm{~m})$, and $(x=2.0 \mathrm{~m} ; y=2.0 \mathrm{~m})$, equally spaced $0.1 \mathrm{~m}$ along the $\mathrm{x}$ and $\mathrm{y}$ directions. The pressure response is computed over this grid of receivers for frequencies of $100 \mathrm{~Hz}, 1000 \mathrm{~Hz}$ and $2000 \mathrm{~Hz}$, using both the 
analytical and the numerical strategies. An average relative error was then calculated as

$$
\overline{\mathrm{E}}=\sum_{i=1}^{\text {Nrec }} \frac{\left|p_{i}^{\text {analytical }}-p_{i}^{\text {numerical }}\right|}{\left|p_{i}^{\text {analytical }}\right|}
$$

This equation allows assessing the global accuracy of the numerical model. The average error computed for the three frequencies using this approach is depicted in fig. $3 \mathrm{~b}$, for the case in which the 75 boundary nodes (and boundary elements) are located around the interface between the two fluids, and 376 internal collocation points are uniformly distributed within the circular inclusion.

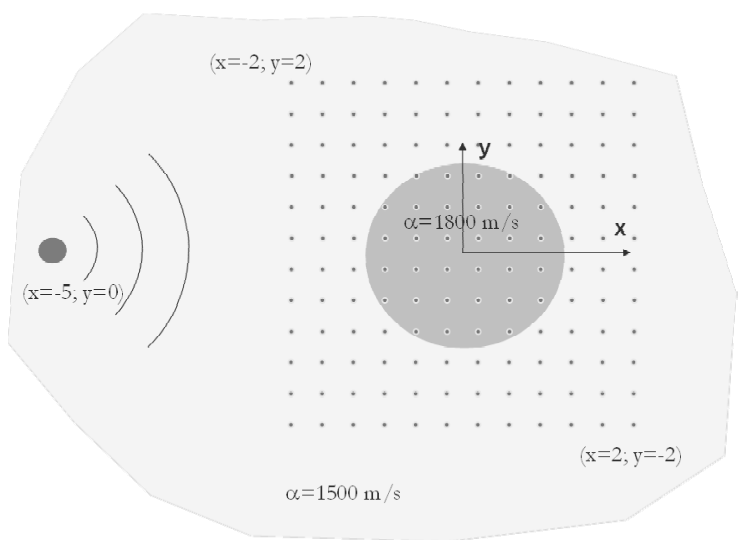

a)

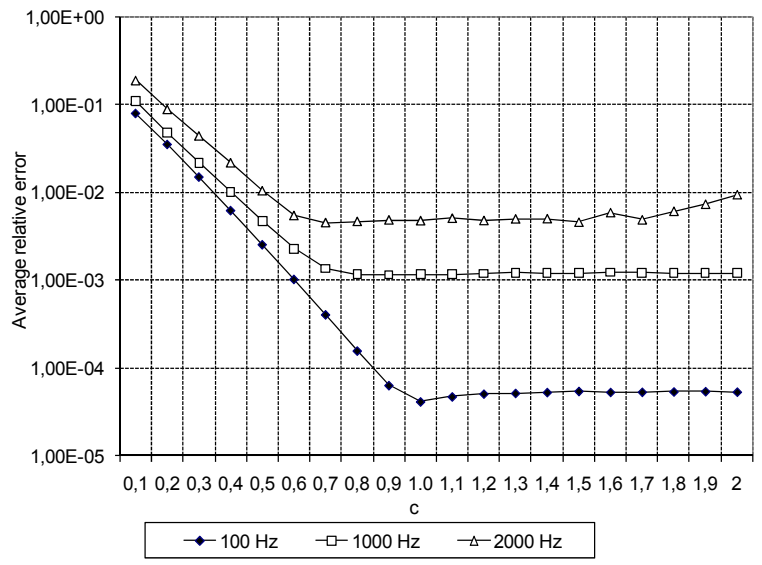

b)

Figure 3: a) Problem geometry; b) average relative error ( $\overline{\mathrm{E}}$ ) for varying values of the free parameter when 75 boundary nodes are used. 
In that plot, values of the free RBF parameter ranging from 0.1 to 2.0 are considered. For Kansa's method, it is well known that the accuracy of the method depends on the correct choice of the free parameter; the same behavior can be observed here, with the average error varying as much as three orders of magnitude with variation of c. Clearly, an increase in this parameter originates a better response, up to a point that the average error becomes almost constant. Interestingly, the lower error level is obtained for a relatively broad range of values of c. As expected, the error obtained using this point distribution is considerably lower for the lower frequency, and then increases as smaller wavelengths are considered.

Although the evaluation of the error is straightforward for this specific configuration, it can become much more difficult for more general cases, for which it is no longer possible to obtain a reference solution analytically. Thus it is important to devise a general strategy that allows a quantification of the global error, without relying on the previous knowledge of an analytical solution. For this purpose, it is interesting to observe that the system of equations defined for Kansa's method imposes that the governing PDE is satisfied at a discrete set of internal points (collocation points), but it does not enforce its satisfaction in the remaining portion of the domain. To illustrate this behaviour, consider the point distribution in fig. $4 \mathrm{a}$, in which 40 nodes are located along the boundary, and 101 internal collocation points are uniformly distributed within the domain. Fig. 4b illustrates the evaluation of

$$
\varepsilon=\nabla^{2} \hat{p}(\mathrm{x})+\frac{\omega^{2}}{\alpha(\mathrm{x})^{2}} \hat{p}(\mathrm{x})
$$

throughout the internal fluid domain. Clearly, in this plot, the value of $\varepsilon$ gets very small (tending to zero) at the collocation points, but becomes considerably larger at other domain points. Considering a number $N_{r e c 2}$ of internal domain points, an average value of $\varepsilon$ can be calculated as

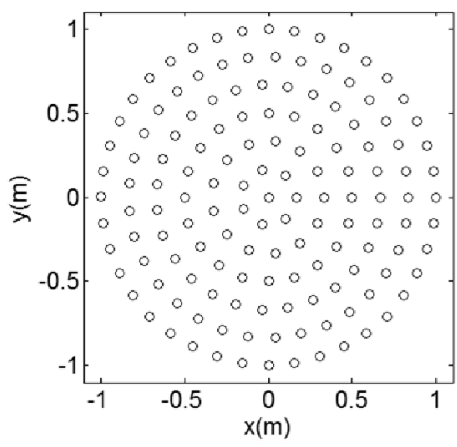

a)

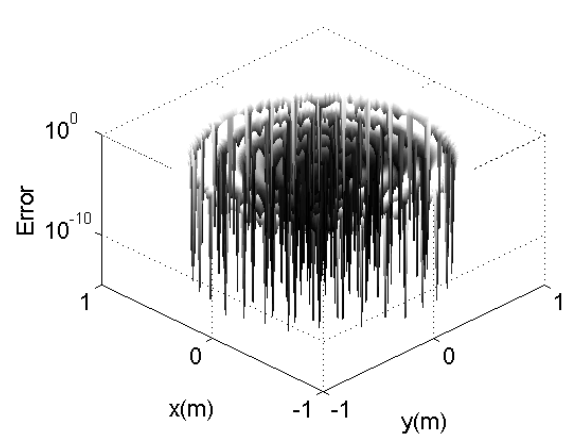

b)

Figure 4: a) Node distribution using 40 boundary nodes; b) spatial distribution of $\varepsilon$ (calculated from eqn. 13) when $\mathrm{c}=0.5$. 


$$
\bar{\varepsilon}=\sum_{i}^{N_{r e c 2}} \frac{\left|\nabla^{2} \hat{p}\left(\mathrm{x}_{i}\right)+\frac{\omega^{2}}{\alpha\left(\mathrm{x}_{i}\right)^{2}} \hat{p}\left(\mathrm{x}_{i}\right)\right|}{N_{\text {rec } 2}}
$$

where $\mathrm{x}_{i}$ is the $i^{\text {th }}$ domain point.

Eqn. 14 has been applied to evaluate the domain error when analyzing the three frequencies defined above, and considering the same problem defined in fig. 3a. Both this domain error and the average error with relation to the analytical solution ( $\overline{\mathrm{E}}$, from eqn. (12)) were computed for a full range of values of the parameter $\mathrm{c}$, and for varying numbers of collocation points and boundary elements. Fig. 5 illustrates the computed values of both parameters, as a function

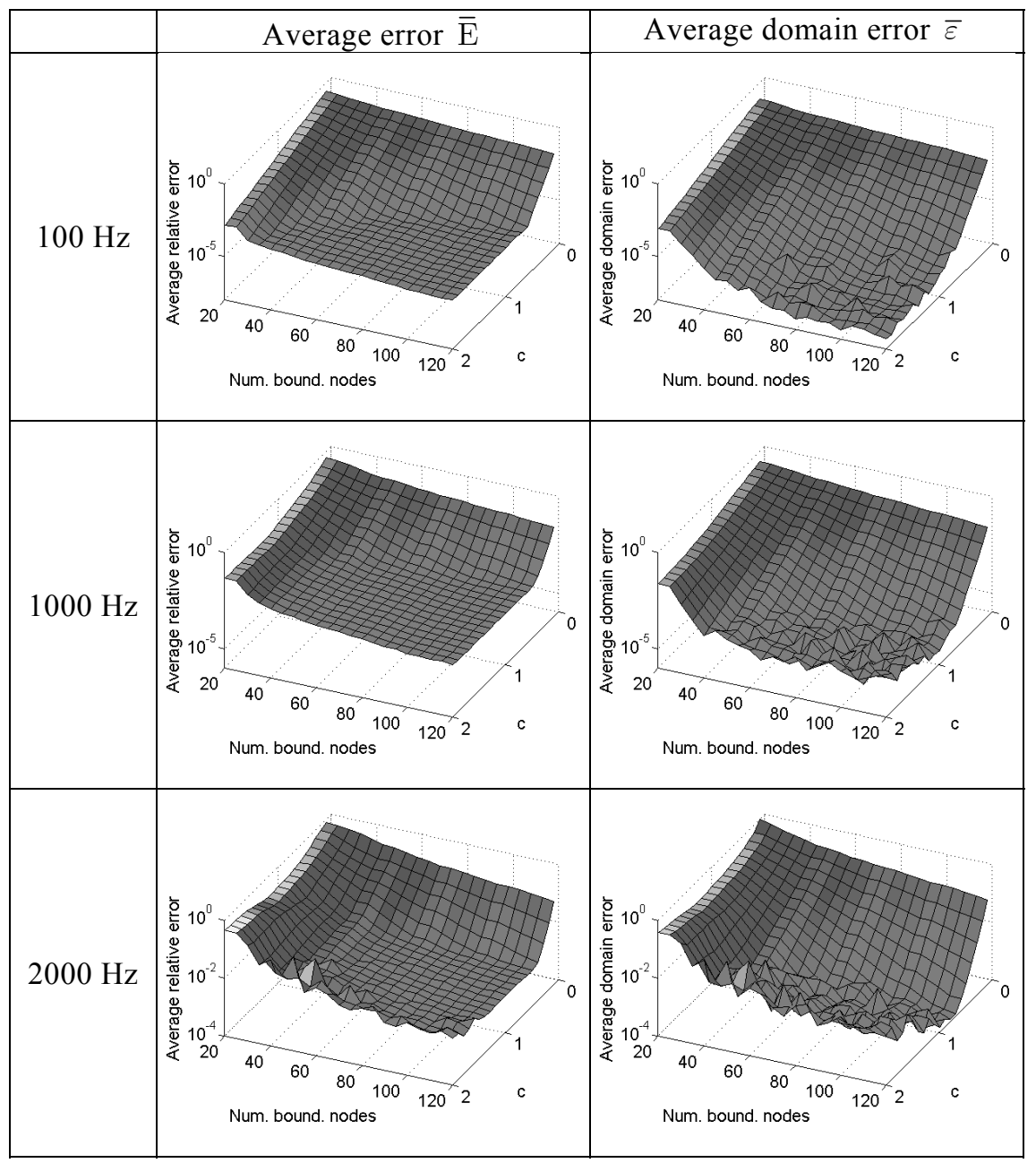

Figure 5: Evaluation of the error using the two methodologies. 
of the free parameter and of the number of boundary nodes. Observing the plots in this figure, for the three analyzed frequencies, it becomes clear that there is a strong relation between both parameters. In fact, observing the error plots computed in terms of $\overline{\mathrm{E}}$, for each value of $\mathrm{c}$, the response initially improves sharply when the number of collocation points is increased; then, for larger numbers of collocation points, the improvement becomes much less pronounced, and a more constant behaviour is observed. Similarly, for a given number of points, the progressive increase of the free parameter originates an improvement in the accuracy, up to a point that the error becomes approximatelly constant.

When the plots computed using the parameter $\bar{\varepsilon}$ are observed, a very similar behavior can be identified, indicating a strong relation between the two error evaluation strategies. It should, however, be noticed that, for the parameter $\bar{\varepsilon}$, visible oscillations can be seen in these plots when larger values of $c$ are used together with larger numbers of boundary points. These oscillations correspond to a numerical instability of Kansa's method, which is well known from previously published works by several authors (see, e.g., Kansa and Hon [10]).

The behaviour observed in the presented results allows inferring that, since a close relation can be seen between the two error estimates, the parameter $\bar{\varepsilon}$ may be used as a reliable guideline for the definition of an accurate response for the Kansa's method. Thus, the authors suggest that the definition of a good estimate of the RBF free parameter can be done by progressively increasing its value, up to the point that the parameter $\bar{\varepsilon}$ begins to evidence an oscillatory behavior; given the presented results, a good choice of this parameter for a given distribution of collocation points, corresponds to the first local minimum of $\bar{\varepsilon}$, when it is written as a function of $\mathrm{c}$.

\section{Final remarks}

A coupled numerical strategy, making use of Kansa's method and of the Boundary Element Method, has been presented in this work for the case of frequency domain acoustic wave scattering. Although the results presented here refer to piecewise constant media, the joint use of both methods can be advantageous whenever a heterogeneous region with spatially varying properties exists within the domain. Since the Kansa's method makes use of RBFs, which may include a variable parameter, a general strategy for the definition of such parameter has been devised, based on the evaluation of the differential operator from the governing PDE throughout the domain. The presented results have shown a close relation between the error evaluated using this strategy and the error computed with relation to a closed form solution.

\section{Acknowledgements}

The authors acknowledge the "Instituto de Investigação e Desenvolvimento Tecnológico em Ciências da Construção - ITeCons", for the support provided during the development of this work. 


\section{References}

[1] Wu, T., Boundary element acoustics, WIT Press, Southampton, UK, 2000.

[2] Tadeu, A., António, J., Amado Mendes, P. and Godinho, L., Sound pressure level attenuation provided by thin rigid screens coupled to tall buildings. Journal of Sound and Vibration, 304, pp. 479-496, 2007.

[3] Ji, Z.L., Boundary element analysis of a straight-through hybrid silencer. Journal of Sound and Vibration, 292, pp. 415-423, 2006.

[4] Kansa, E., Multiquadrics - A scattered data approximation scheme with applications to computational fluid-dynamics - I: surface approximations and partial derivative estimates, Comput. Math. Appl., 19, pp. 127-145, 1990.

[5] Kansa, E., Multiquadrics - A scattered data approximation scheme with applications to computational fluid-dynamics-II solutions to parabolic, hyperbolic and elliptic partial differential equations, Comput. Math. Appl., 19, pp. 147-161, 1990.

[6] Tadeu A., Godinho L. and Chen C.S., Performance of the BEM, MFS, and $\mathrm{RBF}$ collocation method in a $2.5 \mathrm{D}$ wave propagation analysis. Boundary Elements XXVII - Incorporating Electrical Engineering and Electromagnetics, ed. A. Kassab, C.A. Brebbia, E. Divo, D. Poljak, WIT Press, 2005.

[7] Ishimaru, A., Theory and application of wave propagation and scattering in random media. Proceedings of the IEEE, 65(7), pp. 1030-1061, 1977.

[8] Cheng H.-D., Golberg M.A., Kansa E.J. and Zammito G., Exponential convergence and h-c Multiquadric collocation method for partial differential equations. Numer. Meth. Partial Diff. Eq., 19, pp. 571-594, 2003.

[9] Tadeu A., Godinho L. and António J., Benchmark solution for 3D scattering from cylindrical inclusions. Journal of Computational Acoustics, 9, pp. 1311-1328, 2001.

[10] Kansa, E. and Hon, Y., Circumventing the ill-conditioning problem with multiquadric radial basis functions: Applications to elliptic partial differential equations, Computers and Mathematics with Applications, 39, pp. 123-137, 2000. 